\title{
Incidents resulting from staff leaving normal duties to attend medical emergency team calls
}

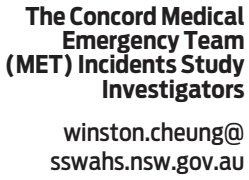

MJA 2014; 201: 528-531 doi: 10.5694/mjal4.00647

\section{C} linical emergency response systems such as medical emergency teams (METs), ${ }_{1}^{1}$ rapid response teams, ${ }^{2}$ patient-at-risk teams ${ }^{3}$ and critical care outreach teams ${ }^{4}$ are now used in hospitals worldwide to manage patients who have unexpected clinical deterioration. Currently, the optimal staffing structure for these systems remains unknown. 5,6

At our hospital, MET personnel are not rostered solely for staffing the MET. Instead, MET staff have normal hospital duties to perform and, when a MET call is activated, they temporarily forgo their normal duties to attend.

This study was instigated after reports of potential adverse events, such as delayed medication dispensing, occurring as a result of staff leaving normal duties to attend MET calls. Our review found no publications in this area. The primary objective was to determine the rate of adverse events and incidents occurring as a result of hospital staff leaving normal duties to attend MET calls.

\section{Methods}

This single-centre, structured interview- and questionnaire-based study was conducted over an 18-week period between 29 July and 15 December 2013. The study was conducted in a 650-bed university teaching hospital in Sydney, New South Wales. Participants were all hospital staff who were recorded as attending a MET call on the hospital campus during the study period.

The primary outcome measure was the rate of adverse events and incidents directly related to MET staff leaving normal duties to attend MET calls. Secondary outcome measures were the rates of such events according to staff occupation.

Our hospital used a two-tiered MET system.7,8 The first tier recommended early clinical review by ward teams, and the second tier activated

\section{Abstract}

Objective: To determine the rate of adverse events and incidents occurring as a result of hospital staff leaving normal duties to attend medical emergency team (MET) calls.

Design, participants and setting: Single-centre, interview and questionnairebased study of staff attending MET calls at a 650-bed university teaching hospital in Sydney, New South Wales, July to December 2013.

Main outcome measure: The rate of adverse events and incidents directly related to MET staff leaving normal duties to attend MET calls.

Results: During the study period, 1490 structured interviews were conducted, and 279 written questionnaires were returned (overall response rate, 66.4\%). There were no adverse events. There were 378 recorded incidents. The incident rate was 213.7 incidents per 1000 MET participant attendances $(95 \% \mathrm{Cl}, 194.8-$ 233.5), and 1.1 incidents per MET call. Using the severity assessment code, $99.5 \%$ of incidents were classified as minimum. The most commonly reported incidents were disruptions to normal duties, ward rounds, and patient reviews. Only $0.8 \%$ of incidents were reported on institutional incident reporting systems.

Conclusion: Significant disruption to normal hospital routines and inconvenience to staff occurred, without causing major harm to patients, when MET staff temporarily left normal duties to attend MET calls. Normal hospital incident reporting systems cannot be used to monitor for these problems, as they are underreported.

the MET. The MET, led by a medical registrar, included an intensive care registrar, an anaesthetic registrar, three residents or interns, a clinical nurse consultant, and nursing staff from the cardiology department. Security and environmental services staff attended MET calls outside of hospital buildings.

All MET staff had normal hospital duties to perform, and would forgo those duties to attend MET calls. MET attendance to MET calls was mandatory.

In 2013, cardiac arrests comprised $4.1 \%$ of MET calls at the hospital.

All staff attending and providing assistance at MET calls had their details recorded on attendance logs. On weekdays after each MET call, trained interviewers would contact the staff listed. Staff who consented were interviewed using a structured interview form. The following data were collected: number of days since the MET call; staff designation; issues resulting from leaving normal duties to attend the MET call; mechanism of reporting, such as line manager or computerised incident reporting system; and self-reported estimated time spent at the MET call.

To avoid intruding on staff when they were not at work, interviewers were instructed to make reasonable attempts to contact staff either in person, or using their hospital pager or phone, during working hours only. Staff who could not be contacted were sent a written questionnaire version of the structured interview. Completion and return of the questionnaire was considered as consent to participate.

Ethics approval was obtained from the hospital's Human Research and Ethics Committee (CH62/6/2013-030).

\section{Study definitions}

The lack of standardised definitions for adverse events was problematic. The National Health and Medical Research Council (NHMRC) did not have definitions for adverse events that were unrelated to pharmaceutical products or medical devices. ${ }^{9}$

The original study definition of adverse event was an "anticipated or unanticipated event that causes, or requires an intervention to prevent, an unfavourable change in a 
person's condition".10,11 Institutional approval for the study to proceed, however, was conditional on altering the definition to that used by NSW Health. ${ }^{12}$ An adverse event was therefore defined as "an unintended patient injury or complication from treatment that results in disability, death or prolonged hospital stay, and is caused by health care management".12 An incident was defined as "any unplanned event resulting in, or with the potential for, injury, damage or other loss".12

Daytime was defined as 08:0015:59, evening as 16:00-11:59, and night-time as midnight to 07:59. The response rate was defined as the number of completed interviews divided by the number of eligible reporting units. ${ }^{13}$

All incidents were classified according to severity assessment $\operatorname{codes}^{12}$ (Appendix 1; online at mja. com.au) by the hospital manager for clinical quality and risk. Incidents were coded as minimum, minor, moderate, major or serious. Incidents that caused no injury or increased level of patient care, which required no additional review, and led to no financial or service losses were coded as minimum. All incidents were reviewed by an independent safety monitor, and managed using normal hospital procedures.

\section{Statistical analysis}

The MET call rate preceding this study was 17.2 MET calls per 1000 admissions. If our study was conducted similarly to a previous study that ran for 131 days and had a response rate of $64.1 \%$, ${ }^{14}$ we predicted that 312 MET calls would occur and 1630 interviews would be completed. This would provide a $95 \%$ confidence interval of $\pm 9.7 \%$ if the primary outcome measure rate was 200 per 1000 MET participant attendances.

Statistical analysis was performed by an independent statistician, using Interactive Statistical Calculation Pages (John C Pezzullo; http://statpages.org/confint. html\#Binomial), and SPSS, version 22 (IBM Corporation). Rates were calculated with binomial 95\% confidence intervals, and subgroups were compared using the Pearson $\chi^{2}$ test, where appropriate.
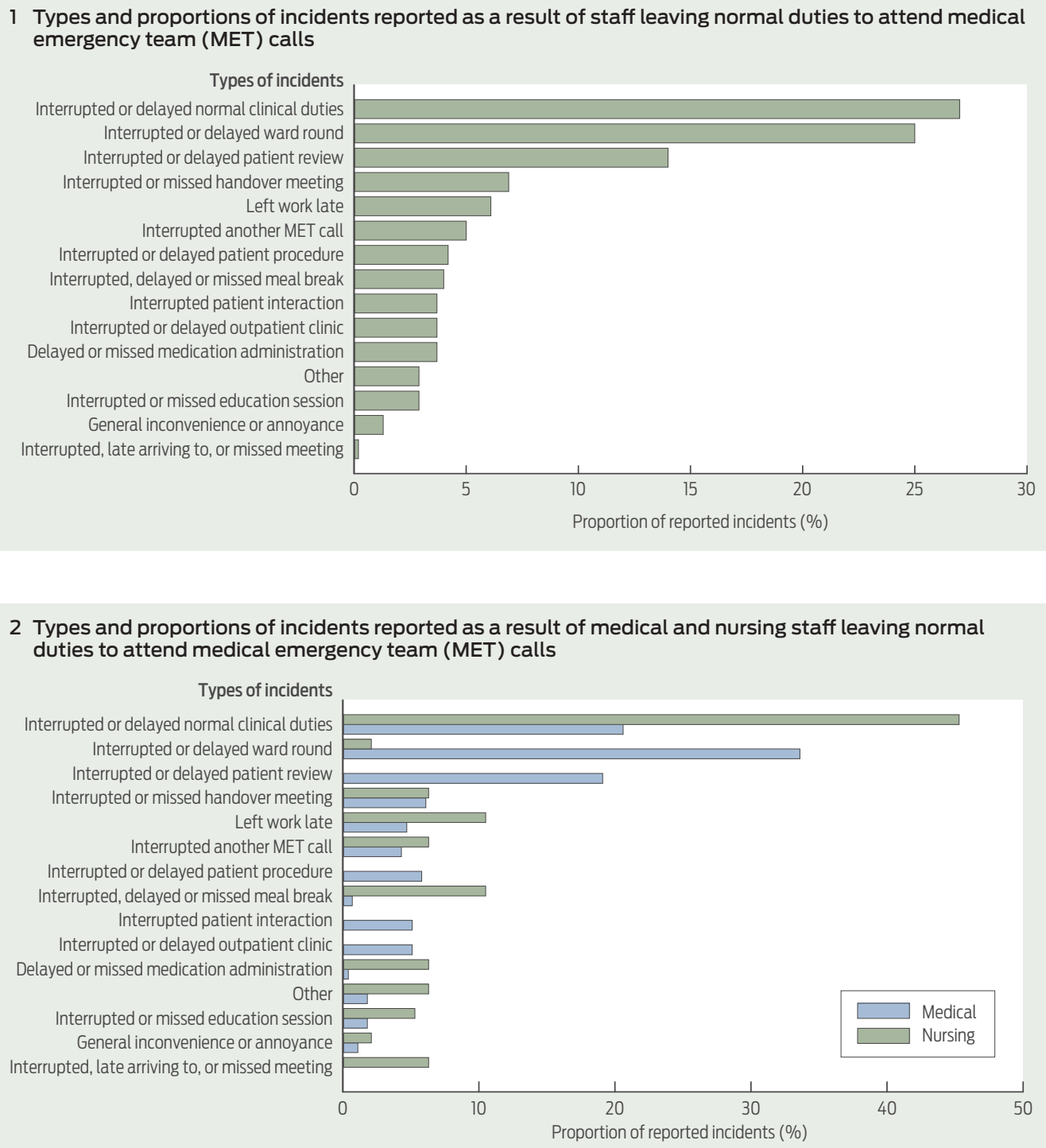

\section{Results}

The hospital admitted 17445 patients in the study period, during which there were 332 MET calls (a mean of 2.6 MET calls per day). The MET call rate was $19.0 \mathrm{MET}$ calls per 1000 admissions (95\% CI, 17.1-21.2).

There were 2663 MET call participant attendances recorded. A mean of eight staff members were recorded at each MET call.

Interviews or questionnaires were completed for 1769 staff, a response rate of $66.4 \%$. Interviewers completed 1490 interviews, and 279 written questionnaires were returned $(84.2 \%$ and $15.8 \%$ of total response, respectively). The median time from MET call to interview and MET call to questionnaire completion was 5 days and 21 days, respectively.
Of staff members participating at MET calls, where staff designation was recorded $(n=2392), 2087$ were MET staff (87.2\%), 289 were ward staff $(12.1 \%)$, and 16 were bystanders $(0.7 \%)$. Of participating staff, where profession was recorded $(n=2405)$ 1545 were medical staff $(64.2 \%)$ and 832 were nursing staff (34.6\%).

There were no adverse events recorded. There were 378 recorded incidents. The incident rate was 213.7 incidents per 1000 MET participant attendances (95\% CI, 194.8-233.5), and 1.1 incidents per MET call.

Using the severity assessment code, there were two incidents $(0.5 \%)$ classified as minor, and 376 incidents $(99.5 \%)$ classified as minimum. There were no incidents classified as serious, major or moderate. 
3 Proportion of incidents, with $95 \%$ confidence intervals, reported as a result of staff leaving normal duties to attend medical emergency team calls, by staff type

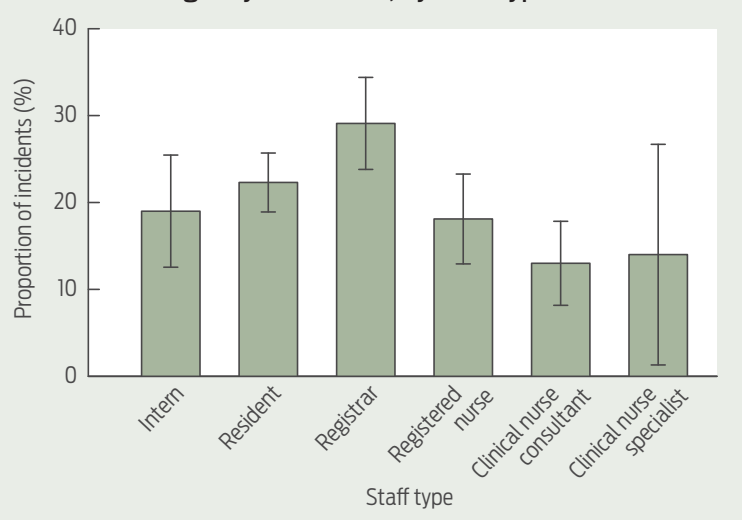

Three incidents $(0.8 \%)$ were reported on institutional incident reporting systems. The types of incidents and the proportions of each are shown in Box 1.

Of the two incidents classified as minor, in the first, a patient absconded from the ward and was subsequently found. In the second, a patient sustained a fall without injury. Both incidents occurred while the patient's nurse had left the ward to attend a MET call.

The incident rate for completed interviews and written questionnaires was 222.1 and 168.5 per 1000 MET participant attendances, respectively $(P=0.045)$.

Medical staff and nursing staff reported 243.0 and 156.8 incidents per 1000 MET participant attendances, respectively $(P<0.001)$. The types of incidents and the proportions of each are shown by role (medical or nursing) in Box 2, and overall proportions by staff type in Box 3 . Most incidents $(127 ; 38.3 \%)$ occurred during daytime hours, 113 in the evening (34.0\%) and 92 during night-time $(27.7 \%)$ (Appendix 2; online at mja.com.au).

The median time spent by staff at MET calls was 20 minutes. The proportion of staff who spent 30 minutes or fewer at a MET call was $74.9 \%$. Staff who spent 60 minutes or longer at the MET call reported significantly more incidents (Appendix 3; online at mja.com.au).

There were 21 occasions $(6.3 \%$ or about once every 6 days) where two MET calls occurred within 30 minutes, and two occasions $(0.6 \%$ or about once every 2 months) where three MET calls occurred within 30 minutes.

\section{Discussion}

This study demonstrated three key findings about when MET staff temporarily left normal duties to attend MET calls. First, no major patient harm occurred. Second, MET calls caused significant disruption to normal hospital routines and inconvenience to staff. This occurred despite most staff spending 30 minutes or less at MET calls. Third, problems that did occur were significantly underreported using normal hospital reporting systems.

The observation that medical staff reported more incidents than nursing staff is consistent with work arrangements. Ward nursing staff provide cover when fellow staff members are indisposed. Medical staff and specialist nursing staff are less likely to have cover because of the specialised nature of their work. Improving cover if MET duty is predicted to affect activities such as procedures, clinics, ward rounds or meal breaks may reduce disruption.

Reducing disruption could also be achieved by reducing the number of junior MET staff and adding a further tier to the MET system, where a smaller MET attends middle-tier MET calls. This would work best in hospitals where the cardiac arrest rates are low. Superfluous staff should also be dismissed to normal duties as soon as practical.

Absolving MET staff of normal duties may reduce disruption; however, a standalone MET at our institution was previously not deemed justifiable because of the low MET call rate.

Whether our results can be extrapolated to other hospitals is uncertain. Our MET call rate appears to be low. Other Australian studies document MET call rates of 8.7-71.3 calls per 1000 admissions. ${ }^{15-20}$ Hospitals with different MET call rates or MET configurations are likely to have different incident rates and patterns.

The very low formal incident reporting rate is not unexpected, as conventional reporting systems are not designed to detect the problems that this study examined.
The main strength of our study was the large number of respondents. The response rate was reasonable, given our intention not to intrude on staff recreational time, and difficulties interviewing staff working outside of business hours or part-time.

There did not appear to be a reporting bias with the use of the written questionnaires, as more incidents were reported from direct interviews. However, recall bias may have occurred in participants surveyed using written questionnaires because of time delay.

This is the first study to quantify the problems resulting from staff leaving normal duties to attend MET calls. However, our results cannot be generalised to other institutions due to differences in patient care and MET systems. Future studies are needed to quantify these problems in different MET systems, and also to identify which method of staffing the MET results in the least amount of disruption, while ensuring appropriate patient care and maximising efficiency.

Acknowledgements: We thank Jenny Peat for her help with statistical analysis, Rochelle Facer for her help with the independent safety monitoring, and the hospital staff who participated in the study.

Full author details are available in Appendix 4 (online at mja.com.au).

Competing interests: No relevant disclosures.

Received 5 May 2014, accepted 11 Sep 2014.

1 Lee A, Bishop G, Hillman KM, Daffurn K. The medical emergency team. Anaesth Intensive Care 1995; 23: 183-186.

2 Berwick DM, Calkins DR, McCannon CJ, Hackbarth AD. The 100000 lives campaign: setting a goal and a deadline for improving health care quality. JAMA 2006; 295: 324-327.

3 Goldhill DR, Worthington L, Mulcahy A, et al. The patient-at-risk team: identifying and managing seriously ill ward patients. Anaesthesia 1999: 54: 853-860.

4 Garcea G, Thomasset S, McClelland L, et al. Impact of a critical care outreach team on critical care readmissions and mortality. Acta Anaesthesiol Scand 2004; 48: 1096-1100.

5 Jones DA, DeVita MA, Bellomo R. Rapidresponse teams. N Engl J Med 2011; 365: 139-146.

6 Cheung W, Mann-Farrar J, Gullick J, et al. Future trials to investigate a ward physician leadership model for the Medical Emergency Team are not logistically feasible. Anaesth Intensive Care 2013; 41: 679-680.

7 NSW Health Clinical Excellence Commission. Between the Flags: keeping patients safe. http://www.health.nsw.gov.au/initiatives/btf/ index.asp (accessed Apr 2014).

8 NSW Health. Recognition and management of patients who are clinically deteriorating. Policy directive PD2013_049. 2013. http:// www0.health.nsw.gov.au/policies/pd/2013/ PD2013_049.html (accessed Apr 2014). 
9 National Health and Medical Research Council. NHMRC glossary. http://www.nhmrc.gov.au/ book/glossary (accessed Apr 2014).

10 ClinicalTrials.gov. "Basic Results" data element definitions. Draft. http://prsinfo.clinicaltrials. gov/results_definitions.html (accessed Apr 2014).

11 ClinicalTrials.gov. Glossary of common site terms. http://www.clinicaltrials.gov/ct2/ about-studies/glossary (accessed Apr 2014).

12 NSW Health. Incident management policy. Policy directive PD2007_061. Sydney: NSW Health, July 2007.

13 American Association for Public Opinion Research. Standard definitions: final dispositions of case codes and outcome rates for surveys. 7th ed. 2011. http://aapor.org/
Content/NavigationMenu/AboutAAPOR/ StandardsampEthics/StandardDefinitions/ StandardDefinitions2011.pdf (accessed Jun 2014).

14 Cheung W, Gullick J, Thanakrishnan G, et al. Injuries occurring in hospital staff attending medical emergency team (MET) calls - a prospective, observational study. Resuscitation 2009; 80: 1351-1356.

15 Hillman K, Chen J, Cretikos M, et al; MERIT study investigators. Introduction of the medical emergency team (MET) system: a clusterrandomised controlled trial. Lancet 2005; 365 : 2091-2097.

16 Vetro J, Natarajan DK, Mercer I, et al. Antecedents to cardiac arrests in a hospital equipped with a medical emergency team. Crit Care Resusc 2011; 13: 162-166.

17 Jones D, Bellomo R, DeVita MA. Effectiveness of the Medical Emergency Team: the importance of dose. Crit Care 2009; 13: 313.

18 Santiano N, Young L, Hillman K, et al. Analysis of medical emergency team calls comparing subjective to "objective" call criteria. Resuscitation 2009; 80: 44-49.

19 Casamento AJ, Dunlop C, Jones DA, Duke G. Improving the documentation of medical emergency team reviews. Crit Care Resusc 2008; 10:29.

20 Jones $\mathrm{D}$, Bates $\mathrm{S}$, Warrillow S, et al. Effect of an education programme on the utilization of a medical emergency team in a teaching hospital. Intern Med J 2006; 36: 231-236. 